\title{
HAHN-BANACH TYPE THEOREMS FOR LOCALLY CONVEX CONES
}

\author{
WALTER ROTH \\ (Received 25 September 1998; revised 18 May 1999) \\ Communicated by P. G. Dodds
}

\begin{abstract}
We prove Hahn-Banach type theorems for linear functionals with values in $\mathbb{R} \cup\{+\infty\}$ on ordered cones. Using the concept of locally convex cones, we provide a sandwich theorem involving sub- and superlinear functionals which are allowed to attain infinite values. It renders general versions of well-known extension and separation results. We describe the range of all linear functionals sandwiched between given suband superlinear functionals on an ordered cone. The Iesults are of interest even in vector spaces, since we consider sublinear functionals that may attain the value $+\infty$.
\end{abstract}

1991 Mathematics subject classification (Amer. Math. Soc.): primary 46A22, 46A03, 46A20.

Keywords and phrases: Hahn-Banach type theorems, locally convex cones.

\section{Introduction}

A cone is a set $\mathscr{P}$ endowed with an addition $(a, b) \mapsto a+b$ and a scalar multiplication $(\alpha, a) \mapsto \alpha a$ for real numbers $\alpha \geq 0$. The addition is supposed to be associative and commutative, and there is a neutral element $0 \in \mathscr{P}$. For the scalar multiplication the usual associative and distributive properties hold, that is $\alpha(\beta a)=(\alpha \beta) a,(\alpha+\beta) a=$ $\alpha a+\beta a$, and $\alpha(a+b)=\alpha a+\alpha b$ for all $a, b \in \mathscr{P}$ and $\alpha, \beta \geq 0$. We have $1 a=a$ and $0 a=0$ for all $a \in \mathscr{P}$.

The cancellation law, stating that $a+c=b+c$ implies $a=b$, however, is not required in general. It holds if and only if the cone $\mathscr{P}$ may be embedded into a real vector space.

In addition we assume that $\mathscr{P}$ carries an order, that is a reflexive transitive relation $\leq$ such that $a \leq b$ implies $a+c \leq b+c$ and $\alpha a \leq \alpha b$ for all $a, b, c \in \mathscr{P}$ and $\alpha \geq 0$.

(C) 2000 Australian Mathematical Society 0263-6115/2000 \$A2.00+0.00 
Since equality in $\mathscr{P}$ is obviously such an order, all our results will apply to cones without order structures as well.

A linear functional on a cone $\mathscr{P}$ is a mapping $\mu: \mathscr{P} \rightarrow \overline{\mathbb{B}}=\mathbb{R} \cup\{+\infty\}$ such that $\mu(a+b)=\mu(a)+\mu(b)$ and $\mu(\alpha a)=\alpha \mu(a)$ for all $a, b \in \mathscr{P}$ and $\alpha \geq 0$. In $\overline{\mathbb{R}}$ we consider the usual algebraic operations, in particular $\alpha+\infty=+\infty$ for all $\alpha \in \overline{\mathbb{R}}$, $\alpha(+\infty)=+\infty$ for all $\alpha>0$ and $0(+\infty)=0$. The functional $\mu$ is called monotone if $a \leq b$ implies $\mu(a) \leq \mu(b)$.

In various places the literature deals with linear functionals on cones which take values in $\mathbb{R} \cup\{-\infty\}([4,6])$ instead. The difference between those two points of view is essential and not just a question of reversing the order on $\mathbb{R}$. In vector spaces both approaches coincide, since linear functionals can take only finite values on invertible elements, but in applications for cones the value $+\infty$ arises more naturally. Moreover, allowing sublinear functionals with values in $\overline{\mathbb{R}}$ in connection with Hahn-Banach type theorems leads to more satisfying results which include investigations of hypolinear functionals on vector spaces as in [1].

In Section 2 we formulate a sandwich theorem for ordered cones which improves the result given in [4]. In Section 3 we turn to locally convex cones, which provide topological structures on cones that generalise locally convex topological vector spaces. In this context we prove a generalisation of our first result. Section 4 contains our main extension and separation theorems. In Section 5 we look at the range at a given element of a cone of all linear functionals which are sandwiched between a sub- and a superlinear functional. Our main result generalises the well-known Sup-Inf Theorem $([2,5,7])$ for subharmonicity with respect to a subcone.

\section{A Sandwich Theorem}

In the following let $\mathscr{P}$ be an ordered cone. A sublinear functional on $\mathscr{P}$ is a mapping $p: \mathscr{P} \rightarrow \overline{\mathbb{R}}$ such that

$$
p(\alpha a)=\alpha p(a) \quad \text { and } \quad p(a+b) \leq p(a)+p(b)
$$

holds for all $a, b \in \mathscr{P}$ and $\alpha \geq 0$. Likewise, a superlinear functional on $\mathscr{P}$ is a mapping $q: \mathscr{P} \rightarrow \mathbb{\mathbb { R }}$ such that

$$
q(\alpha a)=\alpha q(a) \quad \text { and } \quad q(a+b) \geq q(a)+q(b)
$$

holds for all $a, b \in \mathscr{P}$ and $\alpha \geq 0$. Note that superlinear functionals can assume only finite values in elements $a \in \mathscr{P}$ such that $-a \in \mathscr{P}$ as well. The following HahnBanach type sandwich theorem is the basis for the duality theory of ordered cones. It improves the result in [4], since we only require the involved sub- and superlinear 
functionals to comply with the order structure in an obvious way. None of them needs to be monotone.

It is convenient to use the pointwise order relation for functions $f, g$ on $\mathscr{P}$; that is we shall write $f \leq g$ to abridge $f(a) \leq g(a)$ for all $a \in \mathscr{P}$.

THEOREM 2.1 (Sandwich Theorem I). Let $\mathscr{P}$ be an ordered cone and let $p: \mathscr{P} \rightarrow$ $\overline{\mathbb{B}}$ be a sublinear and $q: \mathscr{P} \rightarrow \overline{\mathbb{R}}$ a superlinear functional such that

$$
q(a) \leq p(b) \text { whenever } a \leq b \text { for } a, b \in \mathscr{P} \text {. }
$$

There exists a monotone linear functional $\mu: \mathscr{P} \rightarrow \mathbb{\mathbb { R }}$ such that $q \leq \mu \leq p$.

PROOF. We denote by $X$ the set of all monotone sublinear functionals $s: \mathscr{P} \rightarrow \overline{\mathbb{R}}$ such that $q \leq s \leq p$. As the functional

$$
s(a)=\inf \{p(b) \mid a \leq b, b \in \mathscr{P}\} \text { for all } a \in \mathscr{P}
$$

is obviously contained in $X$, we see that $X$ is not empty. We consider the pointwise order on $X$ and apply Zorn's Lemma in order to show that $X$ contains a minimal element: Let $C$ be a totally ordered subset of $X$ and set

$$
s_{0}(a)=\inf \{s(a) \mid s \in C\} \text { for all } a \in \mathscr{P} \text {. }
$$

It is straightforward to check that $s_{0}$ is sublinear, monotone, and that $q(a) \leq s_{0}(a) \leq$ $p(a)$ holds for all $a \in \mathscr{P}$. Therefore $s_{0}$ is a lower bound for $C$ in $X$. Following Zorn's Lemma, $X$ contains a minimal element $\mu$, and all left to show is that $\mu$ is indeed a linear functional: For an arbitrary fixed element $a_{0} \in \mathscr{P}$ set

$$
\alpha_{0}=\sup \left\{q(c)-\mu(b) \mid b, c \in \mathscr{P}, \mu(b)<+\infty, c \leq a_{0}+b\right\}
$$

We observe that for any such $c$ and $b$ as in the definition of $\alpha_{0}$ we have

$$
q(c) \leq \mu(c) \leq \mu\left(a_{0}+b\right) \leq \mu\left(a_{0}\right)+\mu(b),
$$

hence $q(c)-\mu(b) \leq \mu\left(a_{0}\right)$ and $q\left(a_{0}\right) \leq \alpha_{0} \leq \mu\left(a_{0}\right)$. Now we define a functional $\tilde{\mu}$ by

$$
\tilde{\mu}(a)=\inf \left\{\mu(b)+\lambda \alpha_{0} \mid b \in \mathscr{P}, \lambda \geq 0, a \leq b+\lambda a_{0}\right\} .
$$

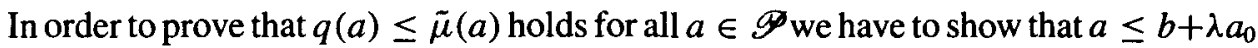
implies $q(a) \leq \mu(b)+\lambda \alpha_{0}$. The latter is obvious if $\mu(b)=+\infty$ or if $\lambda=0$ since $\mu$ is monotone. Otherwise, we have $(a / \lambda) \leq a_{0}+(b / \lambda)$ and by the definition of $\alpha_{0}$

$$
\alpha_{0} \geq q(a / \lambda)-\mu(b / \lambda), \quad \text { or } \quad \lambda \alpha_{0} \geq q(a)-\mu(b) .
$$


Clearly, $\tilde{\mu}$ is sublinear and monotone, and since $\tilde{\mu} \leq \mu$ we conclude that $\tilde{\mu}=\mu$ by the minimality of $\mu$. Thus

$$
\mu\left(a_{0}\right)=\alpha_{0}=\sup \left\{q(c)-\mu(b) \mid b, c \in \mathscr{P}, \mu(b)<+\infty, c \leq a_{0}+b\right\} .
$$

But the mapping $a_{0} \mapsto \alpha_{0}: \mathscr{P} \rightarrow \overline{\mathbb{B}}$ is obviously superlinear. This shows that $\mu$ is linear and completes our proof.

Note that the condition for $q$ and $p$ in Theorem 2.1 is fulfilled if $q \leq p$ and if one of these functionals is monotone. The superlinear functional $q$ however, may not be omitted altogether (or equivalently, replaced by one that allows even the value $-\infty$ ) without further assumptions. We shall demonstrate this in the following example:

EXAMPLE 2.2. Let $\mathscr{P}$ be the vector space of all sequences in $\mathbb{R}$ with only finitely many non-zero elements. For $a=\left(\alpha_{i}\right)_{i \in \mathbb{N}} \in \mathscr{P}$ set $n(a)=\max \left\{i \in \mathbb{N} \mid \alpha_{i} \neq 0\right\}$, if $a \neq 0$ and $n(0)=0$. We define a functional $p$ on $\mathscr{P}$ by

$$
p(a)= \begin{cases}+\infty & \text { if at least one } \alpha_{i}>0, \\ n(a) \sum_{i=1}^{\infty} \alpha_{i} & \text { if } \alpha_{i} \leq 0 \text { for all } i \in \mathbb{N} .\end{cases}
$$

Sublinearity for $p$ is easily checked, and $p$ is even monotone if we consider the canonical (pointwise) order on $\mathscr{P}$. But we do not provide a superlinear functional $q$ as required in the assumption of the preceding Sandwich Theorem 2.1, and we shall show that there is no linear functional $\mu$ on $\mathscr{P}$ which is dominated by $p$ in this case. Assume to the contrary that there is such a functional $\mu$ and set $\varepsilon_{n}=\mu\left(-e_{n}\right)$ where $e_{n}$ denotes the $\mathrm{n}$-th unit sequence in $\mathscr{P}$. For any $n \geq 2$ and $\lambda \geq 0$ set $a_{n}=-\left(\lambda e_{1}+e_{n}\right)$. Then $\mu\left(a_{n}\right)=\lambda \varepsilon_{1}+\varepsilon_{n}$, and as $\mu\left(a_{n}\right) \leq p\left(a_{n}\right)=-n(\lambda+1)$, we conclude that $\lambda \varepsilon_{1}+\varepsilon_{n} \leq-n(\lambda+1)$, that is $\varepsilon_{n} \leq-\lambda\left(\varepsilon_{1}+n\right)-n$. But for an $n>-\varepsilon_{1}$, this can not hold true for all $\lambda \geq 0$.

This example shows that even on a vector space $\mathscr{P}$ for a non-finite sublinear functional $p$ (those functionals are sometimes referred to as hypolinear functionals in the literature, see [1]) there may not be a single linear functional dominated by $p$. Our example may be slightly modified in order to demonstrate that the same may hold true even for a finite sublinear functional $p$ if $\mathscr{P}$ is only a cone. Let $\mathscr{P}$ be the cone of all such sequences from Example 2.2 which have only non-positive components, and define the functional $p$ as above. Then $p$ is finite on $\mathscr{P}$, and the same argument as before shows that there is no $\overline{\mathbb{R}}$-valued linear functional an $\mathscr{P}$ that is dominated by $p$.

For Hahn-Banach type extension and separation results a sandwich-type theorem which allows the functional $q$ to attain the value $-\infty$ clearly seems desirable. The remainder of this paper will be largely devoted to clarify the additional conditions 
which are required in this situation. To prepare our results, let us generalise the notion of a superlinear functional. We set

$$
\overline{\mathbb{R}}=\mathbb{R} \cup\{+\infty,-\infty\}
$$

and further extend the addition and the multiplication by non-negative reals to $\overline{\mathbb{R}}$ through $\alpha+(-\infty)=-\infty$ for all $\alpha \in \overline{\mathbb{R}}$ (thus we set $+\infty+(-\infty)=-\infty$ in particular), $\alpha(-\infty)=-\infty$ for all $\alpha>0$ and $0(-\infty)=0$. The order of $\overline{\mathbb{R}}$ is canonical.

An extended superlinear functional on $\mathscr{P}$ is a mapping $q: \mathscr{P} \rightarrow \overline{\mathbb{B}}$ such that

$$
q(\alpha a)=\alpha q(a) \text { and } q(a+b) \geq q(a)+q(b)
$$

holds for all $a, b \in \mathscr{P}$ and $\alpha \geq 0$. A sandwich theorem involving extended superlinear functionals is best formulated using the concept of locally convex cones.

\section{Neighbourhoods and locally convex cone topologies}

A subcone of an ordered cone $\mathscr{P}$ is a non-empty subset which is closed for addition and multiplication by non-negative scalars.

A subset $\mathscr{C}$ of $\mathscr{P}$ is convex if $\lambda c_{1}+(1-\lambda) c_{2} \in \mathscr{C}$ whenever $c_{1}, c_{2} \in \mathscr{C}$ and $\lambda \in[0,1]$. It is called increasing, or decreasing if $a \in \mathscr{C}$ whenever $c \leq a$, or $a \leq c$ for $a \in \mathscr{P}$ and some $c \in \mathscr{C}$, respectively.

Because subtraction and multiplication by negative scalars are generally not available in a cone, a topological structure should not be expected to be invariant for translation and scalar multiplication. The approach to locally convex cones via convex quasiuniform structures was developed in [5] and uses the following motivation: With every $\overline{\mathbb{R}}$-valued monotone linear functional $\mu$ on $\mathscr{P}$ we may associate a subset

$$
v=\left\{(a, b) \in \mathscr{P}^{2} \mid \mu(a) \leq \mu(b)+1\right\}
$$

of $\mathscr{P}^{2}$ with the following properties:

(U1) $v$ is convex;

(U2) if $a \leq b$ for $a, b \in \mathscr{P}$, then $(a, b) \in v$;

(U3) if $(a, b) \in \lambda v$ and $(b, c) \in \rho v$ for $\lambda, \rho>0$, then $(a, c) \in(\lambda+\rho) v$;

(U4) for every $b \in \mathscr{P}$ there is $\lambda \geq 0$ such that $(0, b) \in \lambda v$.

Any subset $v$ of $\mathscr{P}^{2}$ with the above properties (U1) to (U4) merits being called a uniform neighbourhood for $\mathscr{P}$, and any family $\mathscr{V}$ of such neighbourhoods fulfilling the usual conditions for a quasiuniform structure (see [8]), that is

(U5) for $u, v \in \mathscr{V}$ there is $w \in \mathscr{V}$ such that $w \subset u \cap v$; 
(U6) $\lambda v \in \mathscr{V}$ for all $v \in \mathscr{V}$ and $\lambda>0$,

generates a locally convex cone $(\mathscr{P}, \mathscr{V})$ as elaborated in [5]. More specifically, $\mathscr{V}$ creates three hyperspace topologies on $\mathscr{P}$, and every $v \in \mathscr{V}$ defines neighbourhoods for an element $a \in \mathscr{P}$ by

$$
\begin{aligned}
v(a) & =\{b \in \mathscr{P} \mid(b, a) \in \lambda v \text { for all } \lambda>1\} & & \text { in the upper topology, } \\
(a) v & =\{b \in \mathscr{P} \mid(a, b) \in \lambda v \text { for all } \lambda>1\} & & \text { in the lower topology, } \\
v(a) v & =v(a) \cap(a) v & & \text { in the symmetric topology. }
\end{aligned}
$$

It is convenient to think of a locally convex cone $(\mathscr{P}, \mathscr{V})$ as a subcone of a full locally convex cone $\widetilde{\mathscr{P}}$, that is a cone which contains the neighbourhoods $v$ as positive elements (see [5, Chapter I]). Referring to the order in $\widetilde{\mathscr{P}}$, the relation $a \in v(b)$ may be reformulated as $a \leq b+v$. This leads to a second and equivalent approach to locally convex cones that uses the order structure of a larger full cone in order to describe the topology of $\mathscr{P}$ (see also [8]). Let us indicate how this full cone $\widetilde{\mathscr{P}}$ may be constructed (for details, see [5, Chapter I.5]). For a fixed neighbourhood $v \in \mathscr{V}$ set

$$
\widetilde{\mathscr{P}}=\{a \oplus \alpha v \mid a \in \mathscr{P}, 0 \leq \alpha \in \mathbb{R}\} .
$$

We use the obvious algebraic operations on $\widetilde{\mathscr{P}}$ and the order

$$
a \oplus \alpha v \leq b \oplus \beta v
$$

if either $\alpha=\beta$ and $a \leq b$, or $\alpha<\beta$ and $(a, b) \in \lambda v$ for all $\lambda>\beta-\alpha$. The embedding $a \mapsto a \oplus 0 v$ preserves the algebraic operations and the order of $\mathscr{P}$. The procedure for embedding a locally convex cone $(\mathscr{P}, \mathscr{V})$ into a full cone $(\widetilde{P}, \mathscr{V})$ containing a whole system $\mathscr{V}$ of neighbourhoods as positive elements is similar and elaborated in [5, Chapter I.5].

In the following we shall use this order theoretical approach. We may always assume that a given locally convex cone $(\mathscr{P}, \mathscr{V})$ is a subcone of a full locally convex cone $(\widetilde{P}, \mathscr{V})$ that contains all neighbourhoods as positive elements, and we shall use the order of the latter to describe the topology of $\mathscr{P}$. The above conditions (U1) to (U6) for the quasiuniform structure on $\mathscr{P}$ transfer into equivalent conditions involving the order relation of $\widetilde{P}$ as follows:

(V1) $0 \leq v$ for all $v \in \mathscr{V}$;

(V2) for $u, v \in \mathscr{V}$ there is $w \in \mathscr{V}$ such that $w \leq u$ and $w \leq v$;

(V3) $\lambda v \in \mathscr{V}$ whenever $v \in \mathscr{V}$ and $\lambda>0$;

(V4) for $v \in \mathscr{V}$ and every $a \in \mathscr{P}$ there is $\lambda \geq 0$ such that $0 \leq a+\lambda v$.

Condition (V4) states that every element $a \in \mathscr{P}$ is bounded below. The quasiuniform structure of $\mathscr{P}$ is generated by the subsets

$$
\tilde{v}=\left\{(a, b) \in \mathscr{P}^{2} \mid a \leq b+v\right\} \subset \mathscr{P}^{2}
$$


corresponding to the neighbourhoods $v \in \mathscr{V}$.

Standard examples for locally convex cones include the extended real line $\overline{\mathbb{R}}$ (the neighbourhoods are the strictly positive real numbers), cones of convex subsets of a given locally convex topological vector space (set inclusion defines the order, the neighbourhoods are neighbourhoods of the origin in the given space), or cones of $\mathbb{\mathbb { R }}$-valued functions with various choices for the neighbourhood systems. These and related examples are elaborated in detail in [5, Chapter I].

A linear (sublinear, superlinear) functional $\mu$ on $\mathscr{P}$ is said to be (uniformly) continuous with respect to a neighbourhood $v$ if

$$
\mu(a) \leq \mu(b)+1 \text { holds whenever } a \leq b+v .
$$

Uniform continuity implies monotonicity and continuity with respect to the upper, lower and symmetric topologies on $\mathscr{P}$ and $\overline{\mathbb{R}}$.

The set of all uniformly continuous linear functionals $\mu: \mathscr{P} \rightarrow \mathbb{\mathbb { R }}$ with respect to a certain neighbourhood $v \in \mathscr{V}$ is called the polar $v^{\circ}$ of $v$. The union of all polars, that is the set of all linear functionals that are continuous with respect to some neighbourhood $v \in \mathscr{V}$, is the dual cone $\mathscr{P} *$ of $\mathscr{P}$. It is endowed with the pointwise algebraic operations and the topology $w\left(\mathscr{P}^{*}, \mathscr{P}\right)$ of pointwise convergence of the elements of $\mathscr{P}$, considered as functions ort $\mathscr{P}^{*}$ with values in $\overline{\mathbb{B}}$ with its usual topology. The polar of a neighbourhood is seen to be $w\left(\mathscr{P}^{*}, \mathscr{P}\right)$-compact and convex ([5, Theorem II.2.4]).

We are now ready to formulate a generalised sandwich theorem.

THEOREM 3.1 (Sandwich Theorem II). Let $(\mathscr{P}, \mathscr{V})$ be a locally convex cone, and let $v \in \mathscr{V}$. For a sublinear functional $p: \mathscr{P} \rightarrow \mathbb{\mathbb { R }}$ and an extended superlinear functional $q: \mathscr{P} \rightarrow \overline{\mathbb{B}}$ there exists a linear functional $\mu \in v^{\circ}$ such that $q \leq \mu \leq p$ if and only if

$$
q(a) \leq p(b)+1 \text { whenever } a \leq b+v .
$$

PROOF. The necessity of the condition for $p$ and $q$ is evident, as for any linear functional $\mu \in v^{\circ}$ such that $q \leq \mu \leq p$, and for $a \leq b+v$ we infer from the definition of $v^{\circ}$ that

$$
q(a) \leq \mu(a) \leq \mu(b)+1 \leq p(b)+1 .
$$

For the converse, we assume that our condition for $p$ and $q$ holds for the neighbourhood $v \in \mathscr{V}$ and use the full cone $\widetilde{\mathscr{P}}=\{a \oplus \alpha v \mid a \in \mathscr{P}, 0 \leq \alpha \in \mathbb{R}\}$ from above. We extend the sublinear functional $p$ to $\widetilde{\mathscr{P}}$ setting

$$
\tilde{p}(a \oplus \alpha v)=p(a)+\alpha
$$


and define $\tilde{q}$ on $\widetilde{P}$ by

$$
\tilde{q}(a \oplus \alpha v)=\sup \{q(d)-\lambda \mid d \in \mathscr{P}, \lambda>0 \text { and } d \leq a+\lambda v\}+\alpha .
$$

As $0 \leq a+\lambda v$ for some $\lambda \geq 0$ by (U4), we observe that

$$
\tilde{q}(a \oplus \alpha v) \geq-\lambda+\alpha>-\infty .
$$

It is straightforward to check that $\tilde{q}$ is superlinear. In order to apply our Sandwich Theorem I for the functionals $\tilde{p}$ and $\tilde{q}$ on the ordered cone $\widetilde{P}$ we need to show that

$$
a \oplus \alpha v \leq b \oplus \beta v \text { implies } \quad \tilde{q}(a \oplus \alpha v) \leq \tilde{p}(b \oplus \beta v) .
$$

We argue as follows. By definition of the order on $\widetilde{\mathscr{P}}$ we have either $\alpha=\beta$ and $a \leq b$, or $\alpha<\beta$ and $a \leq b+(\beta-\alpha) v$. For any $d \leq a+\lambda v$ this shows $d \leq b+(\lambda+\beta-\alpha) v$, hence $q(d) \leq p(b)+(\lambda+\beta-\alpha)$ and $(q(d)-\lambda)+\alpha \leq p(b)+\beta$, thus

$$
\tilde{q}(a \oplus \alpha v) \leq \dot{p}(b)+\beta=\tilde{p}(b \oplus \beta v) .
$$

So there is indeed a monotone linear functional $\tilde{\mu}$ on $\widetilde{\mathscr{P}}$ such that $\tilde{q} \leq \tilde{\mu} \leq \tilde{p}$. As $\tilde{\mu}(v) \leq \tilde{p}(v)=1$, we realise that the restriction $\mu$ of $\tilde{\mu}$ to the subcone $\mathscr{P}$ is in the polar of $v$. Finally, $q(a) \leq \tilde{q}(a)$ and $p(a)=\tilde{p}(a)$ holds for all $a \in \mathscr{P}$, hence $\mu$ satisfies the required properties.

An interesting special case occurs if we choose the extended superlinear functional as $q(0)=0$ and $q(a)=-\infty$ for all $0 \neq a \in \mathscr{P}$. Theorem 3.1 states that for a sublinear functional $p$ on a locally convex cone $(\mathscr{P}, \mathscr{V})$ and for $v \in \mathscr{V}$ there is $\mu \in v^{\circ}$ such that $\mu \leq p$ if and only if $p$ is bounded below by -1 on the lower neighbourhood (0) $v$. For locally convex vector spaces this result is due to [1].

A convex subset $\mathscr{L}$ of $\mathscr{P}$ is said to be left-absorbing if it contains $0 \in \mathscr{P}$ and if for every $a \in \mathscr{P}$ there is $l \in \mathscr{L}$ and $\lambda \geq 0$ such that $\lambda l \leq a$. Every left-absorbing set $\mathscr{L}$ gives rise to a neighbourhood $\mathscr{L} v$ defined for elements $a, b \in \mathscr{P}$ by

$$
a \leq b+\mathscr{L} v \quad \text { if } a+l \leq b \text { for some } l \in \mathscr{L} .
$$

Note that for a neighbourhood $v \in \mathscr{V}$ of a locally convex cone $(\mathscr{P}, \mathscr{V})$, the lower neighbourhood (0) $v$ of $0 \in \mathscr{P}$ is increasing and left-absorbing by (V4), and that $a \leq b+{ }_{(0) v} v$ implies $a \leq b+v$. If $\mathscr{P}$ is a vector space, then the reverse also holds true, that is both $v$ and ${ }_{(0) v} v$ define the same neighbourhoods. However, the former observation demonstrates that the neighbourhoods $\mathscr{L} v$ form a basis for the finest locally convex topology on an ordered cone $\mathscr{P}$. In particular, every monotone linear functional on $\mathscr{P}$ is uniformly continuous with respect to some neighbourhood of this type. We shall employ this fact to formulate an algebraic version of Theorem 3.1. 
THEOREM 3.2. Let $\mathscr{P}$ be an ordered cone. For a sublinear functional $p: \mathscr{P} \rightarrow \overline{\mathbb{R}}$ and an extended superlinear functional $q: \mathscr{P} \rightarrow \overline{\mathbb{R}}$ there exists a monotone linear functional $\mu: \mathscr{P} \rightarrow \overline{\mathbb{B}}$ such that $q \leq \mu \leq p$ if and only if there is a left-absorbing convex subset $\mathscr{L}$ of $\mathscr{P}$ such that

$$
q(a) \leq p(b)+1 \text { whenever } a+l \leq b
$$

for $a, b \in \mathscr{P}$ and some $l \in \mathscr{L}$.

If again we choose the extended superlinear functional as $q(0)=0$ and $q(a)=-\infty$ for all $0 \neq a \in \mathscr{P}$, Theorem 3.2 states that for a sublinear functional $p$ on an ordered cone $\mathscr{P}$ there is a monotone linear functional $\mu$ such that $\mu \leq p$ if and only if $p$ is bounded below on an increasing left-absorbing subset of $\mathscr{P}$. Choose the leftabsorbing set $\mathscr{L}$ as in the theorem, and replace it by $\widetilde{L}=\{\tilde{l} \in \mathscr{P} \mid l \leq \tilde{l}$ for $l \in \mathscr{L}\}$, which is increasing and again left-absorbing.

If for every $a \in \mathscr{P}$ there are $c, d \in \mathscr{P}$ with $q(c)>-\infty$ and $p(d)<+\infty$ such that $c \leq a+d$, then we may choose the left-absorbing set

$$
\mathscr{L}=\{l \in \mathscr{P} \mid c \leq l+d \quad \text { for } c, d \in \mathscr{P} \text { with } p(d) \in \mathbb{R}, p(d) \leq q(c)+1\} .
$$

With this insertion, the requirement on $p$ and $q$ in Theorem 3.2 reduces to

$$
q(a) \leq p(b) \text { whenever } a \leq b
$$

for $a, b \in \mathscr{P}$. This situation arises in particular if the superlinear functional $q$ is not extended (choose $c=a$ and $d=0$ in the above condition), or if $\mathscr{P}$ is a vector space and $p$ is finite (choose $c=0$ and $d=-a$ ). Theorem 3.2 thus is seen to recover our first Sandwich Theorem 2.1, as well as the classical sandwich version of the Hahn-Banach theorem for vector spaces.

The following corollary to Theorem 3.1 concerns the case that the sublinear functional $p$ is continuous with respect to some neighbourhood of a locally convex cone. It generalises Theorem II.2.8 from [5].

COROLlaRY 3.3. Let $(\mathscr{P}, \mathscr{V})$ be a locally convex cone, and let $v \in \mathscr{V}$. For a sublinear functional $p: \mathscr{P} \rightarrow \overline{\mathbb{R}}$ the following are equivalent:

(i) $p$ is continuous with respect to $v$;

(ii) for every extended superlinear functional $q: \mathscr{P} \rightarrow \overline{\mathbb{B}}$ such that $q \leq p$ there exists a monotone linear functional $\mu \in v^{\circ}$ such that $q \leq \mu \leq p$;

(iii) $p(a)=\max \left\{\mu(a) \mid \mu \in v^{\circ}\right.$ and $\left.\mu \leq p\right\}$ for all $a \in \mathscr{P}$.

PROOF. If (i) holds and if $q$ is an extended superlinear functional such that $q \leq p$, then for any $a \leq b+v$ we have $q(a) \leq p(a) \leq p(b)+1$ by the continuity of $p$. 
Theorem 3.1 guarantees the existence of a linear functional $\mu$ as in (ii). If, for a given element $a \in \mathscr{P}$, we choose the extended superlinear functional $q$ on $\mathscr{P}$ such that $q(b)=\lambda p(a)$ for $b=\lambda a$ with $\lambda \geq 0$, and $q(b)=-\infty$ otherwise, then it is evident how (iii) follows from (ii). Finally, if (iii) is valid, then for $a \leq b+v$ there is $\mu \in v^{\circ}$ such that $\mu \leq p$ and $\mu(a)=p(a)$. Thus $p(a)=\mu(a) \leq \mu(b)+1 \leq p(b)+1$, and $p$ is continuous with respect to $v$, indeed.

Note that a monotone sublinear functional $p$ on an ordered vector space $\mathscr{P}$ is continuous with respect so some neighbourhood $v$ if and only if it is finitely-valued: Indeed, if $p$ is continuous with respect to $v$, then $0 \leq(-a)+\lambda v$ for every $a \in \mathscr{P}$ and some $\lambda \geq 0$, hence $a \leq \lambda v$ and $p(a) \leq 0+\lambda<+\infty$. If on the other hand $p$ is finitely-valued, choose the left-absorbing convex set $\mathscr{L}=\{l \in \mathscr{P} \mid p(-l) \leq 1\}$, and $p$ is seen to be continuous with respect to $\mathscr{L} v$.

As an application of our last corollary we shall generalise a result for vector spaces from [11]. For a normed space $\mathscr{P}$ with the norm as the sublinear functional $p$ it states the well-known fact that every weakly bounded subset of $\mathscr{P}$ is also norm-bounded. This is usually derived using the Uniform Boundedness Theorem, hence a Baire category argument. Conversely, as shown in [11], our following result can be used to obtain non-Baire proofs of the Uniform Boundedness and Open Mapping Theorems.

THEOREM 3.4. Let $(\mathscr{P}, \mathscr{V})$ be a locally convex cone, and let $v \in \mathscr{V}$. Let $p: \mathscr{P} \rightarrow$ $\overline{\mathbb{R}}$ be a sublinear functional that is continuous with respect to $v$. If $p$ is unbounded on a subset $\mathscr{A}$ of $\mathscr{P}$, then there exists a monotone linear functional $\mu \in v^{\circ}$ such that $\mu \leq p$, and $\mu$ is also unbounded on $\mathscr{A}$.

PROOF. Following Corollary 3.3 there is at least one $\mu \in v^{\circ}$ such that $\mu \leq p$. If $\inf \{p(a) \mid a \in \mathscr{A}\}=-\infty$, then the same holds true for every linear functional $\mu \leq p$, and our claim is obvious. Thus we may assume that $\sup \{p(a) \mid a \in \mathscr{A}\}=+\infty$ and that every functional $\mu \in v^{\circ}$ satisfying $\mu \leq p$ is bounded below on $\mathscr{A}$. We shall show that in this case there is at least one such functional $\mu$ which is unbounded above on $\mathscr{A}$. To this end we construct sequences of elements $a_{n} \in \mathscr{A}$, linear functionals $\mu_{n} \in v^{\circ}$ such that $\mu_{n} \leq p$ and $0<\alpha_{n} \leq 2^{-n}$ as follows.

We set $\alpha_{1}=1 / 2$ and select any $a_{1} \in \mathscr{A}$ such that $p\left(a_{1}\right) \geq 2$. We choose $\mu_{1} \in V^{\circ}$ such that $\mu_{1} \leq p$ and $\mu_{1}\left(a_{1}\right)=p\left(a_{1}\right)$. For $n \geq 2$ we choose $0<\alpha_{n} \leq 2^{-n}$ such that $0 \leq \alpha_{n} a_{i}+2^{-n} v$ for all $i=1, \ldots, n-1$, that is $\alpha_{n} v\left(a_{i}\right) \geq-2^{-n}$ holds for every $v \in v^{\circ}$. Because all the functionals $\mu_{1}, \ldots, \mu_{n-1}$ are bounded below on $\mathscr{A}$ but $\sup \{p(a) \mid a \in \mathscr{A}\}=+\infty$, using Corollary 3.3 we can find $a_{n} \in \mathscr{A}$ and $\mu_{n} \in v^{\circ}$ such that $\mu_{n} \leq p$ and $\sum_{i=1}^{n} \alpha_{i} \mu_{i}\left(a_{n}\right) \geq n$. Finally, we set $\alpha=\sum_{n=1}^{\infty} \alpha_{i} \leq 1$.

For every $c \in \mathscr{P}$ we have $0 \leq c+\lambda v$ for some $\lambda \geq 0$ by (V4), and since all functionals $\mu_{n}$ are contained in $v^{\circ}$ this implies $-\lambda \leq \mu_{n}(c) \leq p(c)$. The series $\mu(c)=(1 / \alpha) \sum_{i=1}^{\infty} \alpha_{i} \dot{\mu}_{i}(c)$ is therefore convergent in $\overline{\mathbb{R}}$ and defines a linear functional 
on $\mathscr{P}$. This is the functional which satisfies our claim: clearly $\mu \leq p$ and $\mu \in v^{\circ}$, since for $c \leq d+v$ we verify that

$$
\mu(c)=\frac{1}{\alpha} \sum_{i=1}^{\infty} \alpha_{i} \mu_{i}(c) \leq \frac{1}{\alpha} \sum_{i=1}^{\infty} \alpha_{i}\left(\mu_{i}(d)+1\right) \leq \mu(d)+1 .
$$

On the other hand, we compute for the elements $a_{n} \in \mathscr{A}$

$$
\alpha \mu\left(a_{n}\right)=\sum_{i=1}^{\infty} \alpha_{i} \mu_{i}\left(a_{n}\right)=\sum_{i=1}^{n} \alpha_{i} \mu_{i}\left(a_{n}\right)+\sum_{i=n+1}^{\infty} \alpha_{i} \mu_{i}\left(a_{n}\right) \geq n-\sum_{i=n+1}^{\infty} 2^{-i} \geq n-1 .
$$

This shows $\sup \{\mu(a) \mid a \in \mathscr{A}\} \geq \sup \left\{\mu\left(a_{n}\right) \mid n \in \mathbb{N}\right\}=+\infty$, thus completing our argument.

A simple example given in [11] shows that an obvious generalisation of our last theorem does not hold true. Even in vector spaces $\mathscr{P}$, and even for a finite sublinear functional $p$ and a subset $\mathscr{A}$ of $\mathscr{P}$, the existence of a linear functional $\mu$ on $\mathscr{P}$ such that $\mu \leq p$ and

$$
\sup \{\mu(a) \mid a \in \mathscr{A}\}=\sup \{p(a) \mid a \in \mathscr{A}\}
$$

is not guaranteed, whether the supremum on the right hand side is finite or infinite.

\section{Extension and separation theorems}

An $\overline{\mathbb{R}}$-valued function $f$ defined on a convex subset $\mathscr{C}$ of an ordered cone $\mathscr{P}$ is called convex if

$$
f\left(\lambda c_{1}+(1-\lambda) c_{2}\right) \leq \lambda f\left(c_{1}\right)+(1-\lambda) f\left(c_{2}\right)
$$

holds for all $c_{1}, c_{2} \in \mathscr{C}$ and $\lambda \in[0,1]$. Likewise, $f: \mathscr{C} \rightarrow \overline{\mathbb{B}}$ is called concave if

$$
f\left(\lambda c_{1}+(1-\lambda) c_{2}\right) \geq \lambda f\left(c_{1}\right)+(1-\lambda) f\left(c_{2}\right)
$$

holds for all $c_{1}, c_{2} \in \mathscr{C}$ and $\lambda \in[0,1]$. An affine function $f: \mathscr{C} \rightarrow \overline{\mathbb{R}}$ is both convex and concave.

Our main extension theorem deals with convex, concave and affine functions on a convex subset $\mathscr{C}$ of $\mathscr{P}$ and yields a variety of known special cases. For functions $f$ and $g$ on $\mathscr{C}$ we shall say that $f \leq g$ on $\mathscr{C}$ if $f(c) \leq g(c)$ for all $c \in \mathscr{C}$.

THEOREM 4.1 (Extension Theorem). Let $(\mathscr{P}, \mathscr{V})$ be a locally convex cone, $\mathscr{C}$ and $\mathscr{D}$ non-empty convex subsets of $\mathscr{P}$, and let $v \in \mathscr{V}$. Let $p: \mathscr{P} \rightarrow \overline{\mathbb{R}}$ be a sublinear and 
$q: \mathscr{P} \rightarrow \overline{\mathbb{R}}$ an extended superlinear functional. For a convex function $f: \mathscr{C} \rightarrow \overline{\mathbb{R}}$ and a concave function $g: \mathscr{D} \rightarrow \overline{\mathbb{R}}$ there exists a monotone linear functional $\mu \in v^{\circ}$ such that

$$
q \leq \mu \leq p, \quad g \leq \mu \quad \text { on } \mathscr{D} \quad \text { and } \mu \leq f \quad \text { on } \mathscr{C}
$$

if and only if

$$
q(a)+\rho g(d) \leq p(b)+\sigma f(c)+1 \text { whenever } a+\rho d \leq b+\sigma c+v
$$

for $a, b \in \mathscr{P}, c \in \mathscr{C}, d \in \mathscr{D}$ and $\rho, \sigma \geq 0$.

PROOF. The necessity of the condition on $p, q, f$ and $g$ for the existence of a functional $\mu \in v^{\circ}$ with the required properties is evident. For the converse implication, assume that our condition holds and define functionals $\tilde{p}$ and $\tilde{q}$ for $x \in \mathscr{P}$ by

$$
\begin{aligned}
& \tilde{p}(x)=\inf \{p(b)+\sigma f(c)+\lambda \mid b \in \mathscr{P}, c \in \mathscr{C}, \lambda, \sigma \geq 0, \text { and } x \leq b+\sigma c+\lambda v\} \\
& \tilde{q}(x)=\sup \{q(a)+\rho g(d) \mid a \in \mathscr{P}, d \in \mathscr{D}, \rho \geq 0, \text { and } a+\rho d \leq x\} .
\end{aligned}
$$

Obviously, $q(x) \leq \tilde{q}(x)$ and $\tilde{p}(x) \leq p(x)$ holds for all $x \in \mathscr{P}$. Furthermore, for all $c \in \mathscr{C}$ and $d \in \mathscr{D}$ we have

$$
\tilde{p}(c) \leq f(c) \text {. and } \tilde{q}(d) \geq g(d) .
$$

For any $x \in \mathscr{P}$ there is by (V4) some $\delta>0$ such that $0 \leq x+\delta v$. Thus, $x \leq b+\sigma c+\lambda v$ for some $b \in \mathscr{P}, c \in \mathscr{C}$ and $\lambda>0, \sigma \geq 0$ implies that $0 \leq b+\sigma c+(\delta+\lambda) v$ and $0 \leq p(b)+\sigma f(c)+(\delta+\lambda)$ by the condition of the theorem, hence $p(b)+\sigma f(c)+\lambda \geq$ $-\delta$, and $\tilde{p}(x) \geq-\delta>-\infty$. The functionals $\tilde{p}$ and $\tilde{q}$ are easily seen to be sublinear respectively extended superlinear on $\mathscr{P}$. All left to show in order to apply Theorem 3.1 with $\tilde{p}$ and $\tilde{q}$ is that $\tilde{q}(x) \leq \tilde{p}(y)+1$ holds for $x, y \in \mathscr{P}$ whenever $x \leq y+v$. Let $x \leq y+v$ and $a, b \in \mathscr{P}, c \in \mathscr{C}, d \in \mathscr{D}, \lambda>0$ and $\rho, \sigma \geq 0$. If $a+\rho d \leq x$ and $y \leq b+\sigma c+\lambda v$, then $a+\rho d \leq b+\sigma c+(1+\lambda) v$ and our condition implies

$$
q(a)+\rho g(d) \leq p(b)+\sigma f(c)+(\lambda+1)
$$

thus $\tilde{q}(x) \leq \tilde{p}(y)+1$. Theorem 3.1 yields the existence of a linear functional $\mu \in v^{\circ}$, and the stated properties follow from $\tilde{q} \leq \mu \leq \tilde{p}$.

If $\mathscr{C}$ (respectively $\mathscr{D}$ ) is even a subcone of $\mathscr{P}$ and if $f$ (respectively $g$ ) is a linear functional, then the condition in Theorem 4.1 obviously needs to be verified only for $\sigma=1$ (respectively $\rho=1$ ). The same will hold for the upcoming Theorem 4.2 and Corollary 4.3. The generality of our Extension Theorem allows a wide range of special cases, a few of which we shall mention. If $g \equiv-\infty$, we have to consider 
the condition of Theorem 4.1 only for $\rho=0$, if $f \equiv+\infty$ only for $\sigma=0$, and if both $g \equiv-\infty$ and $f \equiv+\infty$, then Theorem 4.1 reduces to our previous Sandwich Theorem 3.1. Another case of interest occurs when $\mathscr{C}=\mathscr{D}$ and $f=g$ is an affine function, respectively a linear functional if $\mathscr{C}$ is a subcone of $\mathscr{P}$. For the algebraic case we shall again use the fact that the neighbourhoods generated by left-absorbing subsets form a basis for the finest locally convex topology on an ordered cone.

THEOREM 4.2. Let $\mathscr{P}$ be an ordered cone, $\mathscr{C}$ and $\mathscr{D}$ non-empty convex subsets of $\mathscr{P}$. Let $p: \mathscr{P} \rightarrow \overline{\mathbb{R}}$ be a sublinear and $q: \mathscr{P} \rightarrow \overline{\mathbb{B}}$ an extended superlinear functional. For a convex function $f: \mathscr{C} \rightarrow \overline{\mathbb{R}}$ and a concave function $g: \mathscr{D} \rightarrow \overline{\mathbb{R}}$ there exists a monotone linear functional $\mu: \mathscr{P} \rightarrow \mathbb{\mathbb { R }}$ such that

$$
q \leq \mu \leq p, \quad g \leq \mu \quad \text { on } \mathscr{D} \quad \text { and } \mu \leq f \quad \text { on } \mathscr{C}
$$

if and only if there is a left-absorbing convex subset $\mathscr{L}$ of $\mathscr{P}$ such that

$$
q(a)+\rho g(d) \leq p(b)+\sigma f(c)+1 \text { whenever } a+\rho d+l \leq b+\sigma
$$

for $a, b \in \mathscr{P}, l \in \mathscr{L}, c \in \mathscr{C}, d \in \mathscr{D}$ and $\rho, \sigma \geq 0$.

If for every $a \in \mathscr{P}$ there are $c, d \in \mathscr{P}$ with $q(c)>-\infty$ and $p(d)<+\infty$ such that $c \leq a+d$, then we may again (see the remark following Theorem 3.2) choose the left-absorbing set

$$
\mathscr{L}=\{l \in \mathscr{P} \mid c \leq l+d \text { for } c, d \in \mathscr{P} \text { with } p(d) \in \mathbb{R}, p(d) \leq q(c)+1\} .
$$

With this insertion, the requirement on $p, q, f$ and $g$ in Theorem 4.2 reduces to

$$
q(a)+\rho g(d) \leq p(b)+\sigma f(c) \text { whenever } a+\rho d \leq b+\sigma
$$

for $a, b \in \mathscr{P}, c \in \mathscr{C}, d \in \mathscr{D}$ and $\rho, \sigma \geq 0$. Recall that this situation arises in particular if the superlinear functional $q$ is not extended or if $\mathscr{P}$ is a vector space and $p$ is finite.

The special cases when $q(a)=-\infty$ or when $p(a)=+\infty$ for all $0 \neq a \in \mathscr{P}$ are of particular interest. They may be obtained from Theorem 4.1 by setting $a=0$ or $b=0$, respectively. However, we shall formulate the combination of both cases as another corollary, to yield a generalisation of the Extension Theorem II.2.8 in [5].

COROLLARY 4.3. Let $(\mathscr{P}, \mathscr{V})$ be a locally convex cone, $\mathscr{C}$ and $\mathscr{D}$ non-empty convex subsets of $\mathscr{P}$, and let $v \in \mathscr{V}$. For a convex function $f: \mathscr{C} \rightarrow \mathbb{\mathbb { R }}$ and a concave function $g: \mathscr{D} \rightarrow \overline{\mathbb{B}}$ there exists a monotone linear functional $\mu \in v^{\circ}$ such that

$$
g \leq \mu \quad \text { on } \mathscr{D} \text { and } \mu \leq f \text { on } C
$$


if and only if

$$
\rho g(d) \leq \sigma f(c)+1 \text { whenever } \rho d \leq \sigma c+v
$$

for $c \in \mathscr{C}, d \in \mathscr{D}$ and $\rho, \sigma \geq 0$.

The following result about the separation of convex subsets by a monotone linear functional is another immediate consequence of our Extension Theorem 4.1.

THEOREM 4.4 (Separation Theorem). Let $(\mathscr{P}, \mathscr{V})$ be a locally convex cone, $\mathscr{C}$ and $\mathscr{D}$ non-empty convex subsets of $\mathscr{P}$, and let $v \in \mathscr{V}$. For $\alpha \in \mathbb{R}$ there exists a monotone linear functional $\mu \in v^{\circ}$ such that

$$
\mu(c) \leq \alpha \leq \mu(d) \text { for all } c \in \mathscr{C} \text { and } d \in \mathscr{D}
$$

if and only if

$$
\alpha \rho \leq \alpha \sigma+1 \text { whenever } \rho d \leq \sigma c+v
$$

for $c \in \mathscr{C}, d \in \mathscr{D}$ and $\rho, \sigma \geq 0$.

ProOF. We apply Corollary 4.3 with the functions $f \equiv \alpha$ on $\mathscr{C}$ and $g \equiv \alpha$ on $\mathscr{D}$.

We shall give two immediate corollaries for this separation result.

COROLLARY 4.5. Let $\mathscr{C}$ and $\mathscr{D}$ be disjoint non-empty convex subsets of an ordered cone $\mathscr{P}$, and suppose that for every $a \in \mathscr{P}$ there are $c \in \mathscr{C}, d \in \mathscr{D}$ and $\sigma, \rho \geq 0$ such that $\rho d \leq a+\sigma c$.

(i) If $\mathscr{C}$ is decreasing and $0 \in \mathscr{C}$, then there exists a monotone linear functional $\mu: \mathscr{P} \rightarrow \mathbb{R}$ such that

$$
\mu(c) \leq 1 \leq \mu(d) \text { for all } c \in \mathscr{C} \text { and } d \in \mathscr{D} .
$$

(ii) If $\mathscr{D}$ is increasing and $0 \in \mathscr{D}$, then there exists a monotone linear functional $\mu: \mathscr{P} \rightarrow \overline{\mathbb{B}}$ such that

$$
\mu(c) \leq-1 \leq \mu(d) \text { for all } c \in \mathscr{C} \text { and } d \in \mathscr{D} .
$$

PROOF. We shall use the left-absorbing set

$$
\mathscr{L}=\{l \in \mathscr{P} \mid \rho d \leq l+\sigma c \text { for } c \in \mathscr{C}, d \in \mathscr{D}, \sigma, \rho \geq 0, \sigma+\rho \leq 1\}
$$


and the corresponding neighbourhood $\mathscr{L} v$, that is

$$
a \leq b+\mathscr{L} v \text { if } a+l \leq b \text { for some } l \in \mathscr{L},
$$

in Theorem 4.4. For part (i) we choose $\alpha=1$. Let us assume that, contrary to the condition in Theorem 4.4, $\rho d \leq \sigma c+\mathscr{L} v$ holds for some $c \in \mathscr{C}, d \in \mathscr{D}$ and $\rho>\sigma+1$. Then $\rho d+l \leq \sigma c$ for some $l \in \mathscr{L}$, that is $\rho^{\prime} d^{\prime} \leq l+\sigma^{\prime} c^{\prime}$, hence $\rho d+\rho^{\prime} d^{\prime} \leq \sigma c+\sigma^{\prime} c^{\prime}$ for some $d^{\prime} \in \mathscr{D}, c^{\prime} \in \mathscr{C}$ and $\rho^{\prime}+\sigma^{\prime} \leq 1$. Using the convexity of the sets $\mathscr{C}$ and $\mathscr{D}$ the latter may be reformulated as $\left(\rho+\rho^{\prime}\right) d^{\prime \prime} \leq\left(\sigma+\sigma^{\prime}\right) c^{\prime \prime}$ for some $d^{\prime \prime} \in \mathscr{D}$ and $c^{\prime \prime} \in \mathscr{C}$. Since $\sigma+\sigma^{\prime} \leq \sigma+1<\rho \leq \rho+\rho^{\prime}$, we have $\sigma^{\prime \prime}=\left(\sigma+\sigma^{\prime}\right) /\left(\rho+\rho^{\prime}\right) \leq 1$ and $\sigma^{\prime \prime} c^{\prime \prime} \in \mathscr{C}$. But $d^{\prime \prime} \leq \sigma^{\prime \prime} c^{\prime \prime}$ contradicts our assumption that $\mathscr{C}$ is decreasing and disjoint from $\mathscr{D}$. For part (ii) of the corollary we argue in an analogous way with $\alpha=-1$.

Every element of a locally convex cone $(\mathscr{P}, \mathscr{V})$ is bounded below, that is contained in some multiple of every lower neighbourhood (0) $v$ by (U4) (see Section 3 ). An element $a \in \mathscr{P}$ is called bounded (above) if or every $v \in \mathscr{V}$ there is $\lambda \geq 0$ such that $a \leq \lambda v$, that is $a \in \lambda v(0)$. Bounded elements satisfy a modified version of the cancellation law ([5, Lemma I.4.3]), and continuous linear functionals take only finite values there.

COROLLARY 4.6. Let $\mathscr{B}$ be a non-empty convex subset of a locally convex cone $(\mathscr{P}, \mathscr{V})$ such that $0 \in B$.

(i) If $\mathscr{B}$ is closed with respect to the lower topology on $\mathscr{P}$, then for every element $a \notin \mathscr{B}$ in $\mathscr{P}$ there exists a monotone linear functional $\mu \in \mathscr{P}^{*}$ such that

$$
\mu(b) \leq 1 \leq \mu(a) \text { for all } b \in \mathscr{B},
$$

and indeed $1<\mu(a)$ if a is bounded above.

(ii) If $\mathscr{B}$ is closed with respect to the upper topology on $\mathscr{P}$, then for every element $a \notin \mathscr{B}$ in $\mathscr{P}$ there exists a monotone linear functional $\mu \in \mathscr{P}^{*}$ such that

$$
\mu(a)<-1 \leq \mu(b) \text { for all } b \in \mathscr{B} \text {. }
$$

PROOF. For part (i), since $\mathscr{B}$ is closed with respect to the lower topology (recall the introductory remarks of Section 3 ) on $\mathscr{P}$, there is a lower neighbourhood $(a) u$ such that $(a) u \cap \mathscr{B}=\emptyset$. We shall apply Theorem 4.4 with the neighbourhood $v=(1 / 2) u \in \mathscr{V}$, the convex sets $\mathscr{C}=\mathscr{B}, \mathscr{D}=(a) v$ and $\alpha=1$. Let us assume that, contrary to the condition in Theorem 4.4, for some $b \in \mathscr{B}$ and $a^{\prime} \in(a) v$, that is $a \leq a^{\prime}+v$, there are $\rho>\sigma+1$ such that $\rho a^{\prime} \leq \sigma b+v$. As $0 \in \mathscr{B}$ and $\sigma / \rho<1$, we have $b^{\prime}=(\sigma / \rho) b \in B$ as well. Thus $a^{\prime} \leq b^{\prime}+(1 / \rho) v \leq b^{\prime}+v$, as $1 / \rho<1$. 
Now $a \leq a^{\prime}+v$ yields $a \leq b^{\prime}+2 v$, hence $b^{\prime} \in(a) v$, contradicting our assumption. Theorem 4.4 therefore guarantees that there is $\mu \in v^{\circ}$ satisfying

$$
\mu(b) \leq 1 \leq \mu\left(a^{\prime}\right) \quad \text { for all } b \in \mathscr{B} \text { and } a^{\prime} \in(a) v .
$$

This proves the first part of our statement in (i). If the element $a$ is even bounded above, then there is $\varepsilon>0$ such that $\varepsilon a \leq v$, hence $(1+\varepsilon) a \leq a+v$, and with $a^{\prime}=(1 /(1+\varepsilon)) a$, we realise that $a \leq a^{\prime}+(1 /(1+\varepsilon)) v \leq a^{\prime}+v$, that is $a^{\prime} \in(a) v$. This shows

$$
1 \leq \mu\left(a^{\prime}\right)=(1 /(1+\varepsilon)) \mu(a), \quad \text { hence } 1<1+\varepsilon \leq \mu(a),
$$

as claimed. An analogous argument holds for case (ii) using upper instead of lower neighbourhoods and the fact that every element of a locally convex cone is bounded below.

\section{The Range Theorem}

In this final section we shall investigate the range of linear functionals that are sandwiched between a given sublinear and an extended superlinear functional on an ordered cone. In vector spaces, every $\mathbb{R}$-valued sublinear functional $p$ is the pointwise supremum of all linear functionals that are dominated by $p$, but this observation holds no longer true in general if $p$ attains the value $+\infty$ or if we consider cones instead of vector spaces (see our Example 2.2).

In the following, let $(\mathscr{P}, \mathscr{V})$ be a locally convex cone, $\mathscr{P}^{*}$ its topological dual. Recall from Theorem 3.1 that for a sublinear functional $p$ and an extended superlinear functional $q$ on $\mathscr{P}$ there is at least one linear functional $\mu \in \mathscr{P}^{*}$ satisfying $q \leq \mu \leq p$ if and only if there is a neighbourhood $v \in \mathscr{V}$ such that

$$
q(a) \leq p(b)+1 \text { whenever } a \leq b+v .
$$

In the following main theorem of this section we shall describe the range at a given element $a \in \mathscr{P}$ of all continuous linear functionals $\mu \in \mathscr{P}^{*}$ sandwiched between $p$ and $q$. As usual, we set inf $\emptyset=+\infty$ and $\sup \emptyset=-\infty$.

THEOREM 5.1 (Range Theorem). Let $(\mathscr{P}, \mathscr{V})$ be a locally convex cone. Let $p$ and $q$ be sublinear and extended superlinear functionals on $\mathscr{P}$ and suppose that there is at least one linear functional $\mu \in \mathscr{P}^{*}$ satisfying $q \leq \mu \leq p$. Then for all $a \in \mathscr{P}$

$$
\sup _{\substack{\mu \in \mathscr{T} \\ \mathcal{M} \leq \mu \leq \rho}} \mu(a)=\sup _{v \in \mathscr{V}} \inf \{p(b)-q(c) \mid b, c \in \mathscr{P}, q(c) \in \mathbb{R}, a+c \leq b+v\},
$$


and for all $a \in \mathscr{P}$ such that $\mu(a)$ is finite for at least one $\mu \in \mathscr{P}^{*}$ satisfying $q \leq \mu \leq p$

$$
\inf _{\substack{\mu \in \mathscr{P}^{*} \\ q \leq \mu \leq p}} \mu(a)=\inf _{v \in \mathscr{Y}} \sup \{q(c)-p(b) \mid b, c \in \mathscr{P}, p(b) \in \mathbb{R}, c \leq a+b+v\} .
$$

ProOF. We use $\alpha$ and $\bar{\alpha}$ to abbreviate the left-hand and the right-hand sides of the first equation, and $\beta$ and $\bar{\beta}$ in the same way for the second one. From the assumptions on $p$ and $q$ in the respective cases we know that $\alpha>-\infty$ and $\beta<+\infty$. Let $\mu \in \mathscr{P}^{*}$ such that $q \leq \mu \leq p$. Given $\varepsilon>0$ there is $v \in \mathscr{V}$ such that $\mu \in \varepsilon v^{\circ}$. Then for $a+c \leq b+v$, with $q(c) \in \mathbb{R}$ we have

$$
\mu(a)+q(c) \leq \mu(a)+\mu(c) \leq \mu(b)+\varepsilon \leq p(b)+\varepsilon,
$$

hence $\mu(a) \leq p(b)-q(c)+\varepsilon$, and

$$
\mu(a) \leq \inf \{p(b)-q(c) \mid b, c \in \mathscr{P}, q(c) \in \mathbb{R}, a+c \leq b+v\}+\varepsilon .
$$

A similar argument yields

$$
\mu(a) \geq \sup \{q(c)-p(b) \mid b, c \in \mathscr{P}, p(b) \in \mathbb{R}, c \leq a+b+v\}+\varepsilon .
$$

But this shows $\bar{\beta} \leq \mu(a) \leq \bar{\alpha}$ and $\bar{\beta} \leq \beta \leq \bar{\alpha} \leq \bar{\alpha}$. We proceed to demonstrate that $\alpha \geq \bar{\alpha}$ also holds. For $\alpha=+\infty$ there is nothing to prove. Thus we may assume that $\alpha \in \mathbb{R}$, and we shall show that

$$
\alpha \geq \inf \{p(b)-q(c) \mid b, c \in \mathscr{P}, q(c) \in \mathbb{R}, a+c \leq b+v\}
$$

holds for all $v \in \mathscr{V}$. Given a fixed neighbourhood $v \in \mathscr{V}$ and $0<\varepsilon \leq 1 / 4$ there is $\mu \in \mathscr{P}^{*}$ such that $q \leq \mu \leq p$ and $\mu(a) \geq \alpha-\varepsilon$. We find a neighbourhood $w \in \mathscr{V}$ such that $\mu \in(1 / 2) w^{\circ}$. We may assume that $w \subset v$. Now we shall use Theorem 4.1 for the convex sets $\mathscr{C}=\mathscr{D}=\{a\}$ and the functions $f \equiv+\infty$ and $g \equiv \alpha+\varepsilon$. Since there is no linear functional $\nu \in \mathscr{P}^{*}$ such that $q \leq \nu \leq p$ and $\nu(a) \geq \alpha+\varepsilon$, the condition in Theorem 4.1 must fail for every neighbourhood in $\mathscr{V}$ and in particular for $w$. There are $b, c \in \mathscr{P}$ and $\rho \geq 0$ such that $c+\rho a \leq b+w$ and

$$
q(c)+\rho(\alpha+\varepsilon)>p(b)+1 .
$$

This implies in particular that $p(b)<+\infty$. Since $\mu \in(1 / 2) w^{\circ}$, we also have

$$
q(c)+\rho(\alpha-\varepsilon) \leq \mu(c)+\rho \mu(a)=\mu(c+\rho a) \leq \mu(b)+(1 / 2) \leq p(b)+(1 / 2),
$$

implying that $q(c) \in \mathbb{R}$. Combining the last two inequalities yields

$$
p(b)+1<p(b)+(1 / 2)+2 \rho \varepsilon, \text { hence } \rho>1 /(4 \varepsilon) \geq 1 .
$$


Then multiplying by $1 / \rho<1$ gives $c+\rho a \leq b+w$, which yields $c^{\prime}+a \leq$ $b^{\prime}+(1 / \rho) w \leq b^{\prime}+w$ with $c^{\prime}=c / \rho$ and $b^{\prime}=b / \rho$. Furthermore, we realise that $p\left(b^{\prime}\right)=(1 / \rho) p(b) \in \mathbb{R}, q\left(c^{\prime}\right)=(1 / \rho) q(c) \in \mathbb{R}$ and

$$
p\left(b^{\prime}\right) \leq p\left(b^{\prime}\right)+(1 / \rho)=(1 / \rho)(p(b)+1)<q\left(c^{\prime}\right)+(\alpha+\varepsilon),
$$

that is $p\left(b^{\prime}\right)-q\left(c^{\prime}\right) \leq \alpha+\varepsilon$. Summarising, we obtain

$$
\begin{aligned}
\alpha+\varepsilon & \geq \inf \{p(b)-q(c) \mid b, c \in \mathscr{P}, q(c) \in \mathbb{R}, a+c \leq b+w\} \\
& \geq \inf \{p(b)-q(c) \mid b, c \in \mathscr{P}, q(c) \in \mathbb{R}, a+c \leq b+v\},
\end{aligned}
$$

which yields the first part of our claim. For the second part, we already established that $\bar{\beta} \leq \beta<+\infty$ holds. The reverse inequality $\beta \leq \bar{\beta}$ is trivial if $\beta=-\infty$. For $\beta \in \mathbb{R}$ it may be verified using Theorem 4.1 in an analogous argument as in the first part of our proof.

The first equation in Theorem 5.1 is not valid in general if we do not require that there is at least one linear functional $\mu \in \mathscr{P}^{*}$ satisfying $q \leq \mu \leq p$. If there is no such $\mu$, then the left-hand side equals $-\infty$, but an inspection of our Example 2.2 shows that the right-hand side need not to take the same value. We endow the vector space $\mathscr{P}$ of finite sequences from Example 2.2 with the equality as order and the neighbourhoods from $l^{\infty}$. For $p$ as in Example 2.2 añd $q(a)=-\infty$ for all $0 \neq a \in \mathscr{P}$ we may check that the right-hand side of the first equation in Theorem 5.1 equals $+\infty$ for all sequences $a=\left(\alpha_{i}\right)_{i \in \mathbb{N}} \in \mathscr{P}$ that have at least one entry $\alpha_{i}>0$. Likewise, we shall demonstrate in Example 5.4 that the existence of a linear functional $\mu \in \mathscr{P}^{*}$ satisfying $q \leq \mu \leq p$ which is finite on the element $a \in \mathscr{P}$ is essential for the validity of the second equation in Theorem 5.1.

Theorem 5.1 lends itself to a variety of special cases, a few of which we shall mention. An algebraic version of our result may be obtained by considering the finest locally convex topology on $\mathscr{P}$, that is the system of all neighbourhoods $\mathscr{L} v$, where $\mathscr{L}$ is any a left-absorbing convex subset of $\mathscr{P}$. Also of interest are the cases when $q(a)=-\infty$, respectively $p(a)=+\infty$ for all $0 \neq a \in \mathscr{P}$. They may be easily obtained from our general result by setting $c=0$ or $b=0$, respectively in the right hand sides of the equations. The expressions in Theroem 5.1 may be simplified in some cases of particular interest. If there is a neighbourhood $w \in \mathscr{V}$ satisfying (wp) for all $a \leq b+w$ there is $w^{\prime} \in \mathscr{P}$ such that $p\left(w^{\prime}\right) \leq 1$ and $a \leq b+w^{\prime}$, then obviously $\mu \leq p$ for a linear functional $\mu \in \mathscr{P}^{*}$ implies that $\mu \in w^{\circ}$, hence the condition $q \leq \mu \leq p$ defines a $w\left(\mathscr{P}^{*}, \mathscr{P}\right)$-compact subset of $\mathscr{P}^{*}$. The infimum and the supremum on the left hand sides of the equations in Theorem 5.1 therefore turn into a maximum and a minimum. The right hand sides may also be simplified. 
Clearly,

$$
\begin{array}{r}
\sup _{v \in \mathcal{Y}} \inf \{p(b)-q(c) \mid b, c \in \mathscr{P}, q(c) \in \mathbb{R}, a+c \leq b+v\} \\
\leq \inf \{p(b)-q(c) \mid b, c \in \mathscr{P}, q(c) \in \mathbb{R}, a+c \leq b\}
\end{array}
$$

holds in any case. If (wp) holds true, given $\varepsilon>0$ and $v \leq \varepsilon w$, for $a+c \leq b+v \leq$ $b+\varepsilon w$ we have $a+c \leq\left(b+w^{\prime}\right)$ with $p\left(w^{\prime}\right) \leq \varepsilon$. Therefore $p\left(b+w^{\prime}\right)-q(c) \leq$ $(p(b)-q(c))+\varepsilon$. This demonstrates the reverse inequality

$$
\begin{aligned}
& \inf \{p(b)-q(c) \mid b, c \in \mathscr{P}, q(c) \in \mathbb{R}, a+c \leq b\} \\
& \quad \leq \sup _{v \in \mathcal{V}} \inf \{p(b)-q(c) \mid b, c \in \mathscr{P}, q(c) \in \mathbb{R}, a+c \leq b+v\} .
\end{aligned}
$$

Similarly the expression in the second equality of Theorem 5.1 may be simplified. Moreover, the above arguments may be repeated if we replace condition (wp) by (wq) for all $a \leq b+w$ there is $w^{\prime \prime} \in \mathscr{P}$ such that $q\left(w^{\prime \prime}\right) \geq-1$ and $a+w^{\prime \prime} \leq b$. Using this, we obtain the following corollary.

COROLLARY 5.2. Let $(\mathscr{P}, \mathscr{V})$ be a locally convex cone, and let $p$ and $q$ be sublinear and extended superlinear functionals on $\mathscr{P}$ such that $q(a) \leq p(b)$ holds whenever $a \leq b$. If either condition (wp) or (wq) holds for a certain neighbourhood $w \in \mathscr{V}$, then

$$
\begin{aligned}
& \max _{\substack{\mu \in \mathscr{P}^{*} \\
q \leq \mu \leq p}} \mu(a)=\inf \{p(b)-q(c) \mid b, c \in \mathscr{P}, q(c) \in \mathbb{R}, a+c \leq b\}, \\
& \min _{\substack{\mu \in \mathscr{G} \\
q \leq \mu \leq p}} \mu(a)=\sup \{q(c)-p(b) \mid b, c \in \mathscr{P}, p(b) \in \mathbb{R}, c \leq a+b\}
\end{aligned}
$$

for all $a \in \mathscr{P}$.

PROOF. We still have to verify the additional assumptions of Theorem 5.1. If $q \leq p$, then each of the conditions (wp) and (wq) guaranties that $q(a) \leq p(b)+1$ holds whenever $a \leq b+w$. Thus there is at least one $\mu \in \mathscr{P}^{*}$ satisfying $q \leq \mu \leq p$. For the second equation, if $\mu(a)=+\infty$ for all such $\mu$, then for $\mathscr{C}=\{a\} \subset \mathscr{P}$ and every $n \in \mathbb{N}$ there is no $\mu \in w^{\circ}$ such that $q \leq \mu \leq p$ and $\mu \leq n$ on $\mathscr{C}$. Following Theorem 4.1, we find $a_{n}, b_{n} \in \mathscr{P}$ and $\sigma_{n} \geq 0$ such that $a_{n} \leq b_{n}+\sigma_{n} a+w$ and $q\left(a_{n}\right)>p\left(b_{n}\right)+\sigma_{n} n+1$. The above shows $\sigma_{n}>0$, and if (wp) holds there are $w_{n}^{\prime} \in \mathscr{P}$ such that $p\left(w_{n}^{\prime}\right) \leq 1$ and $a_{n} \leq b_{n}+\sigma_{n} a+w_{n}^{\prime}$, that is $\left(a_{n} / \sigma_{n}\right) \leq a+\left(b_{n}+w_{n}^{\prime}\right) / \sigma_{n}$. Now

$$
q\left(a_{n} / \sigma_{n}\right)-p\left(\left(b_{n}+w_{n}^{\prime}\right) / \sigma_{n}\right) \geq 1 / \sigma_{n}\left(q\left(a_{n}\right)-p\left(b_{n}\right)-1\right)>n
$$

shows that $\sup \{q(c)-p(b) \mid b, c \in \mathscr{P}, p(b) \in \mathbb{R}, c \leq a+b\}=+\infty$, and the second equality holds also in this case. We use a similar argument with (wq). 
The situation of Corollary 5.2 arises in particular if $\mathscr{P}$ contains a certain neighbourhood $w \in \mathscr{V}$ as an element and if $p(w)<+\infty$ or if $-w \in \mathscr{P}$ and $q(-w)>-\infty$. Also, if $q$ does not attain the value $-\infty$ and if the neighbourhood $w=\mathscr{L} v$, where $\mathscr{L}=\{l \in \mathscr{P} \mid q(l) \geq-1\}$. is contained in $\mathscr{V}$, then (wq) holds. Similarly, if $\mathscr{P}$ is a vector space and if $p$ does not attain the value $+\infty$, we may consider the neighbourhood $w=\mathscr{L} v$, where $\mathscr{L}=\{l \in \mathscr{P} \mid p(-l) \leq 1\}$. If $w \in \mathscr{V}$, then (wp) holds. As an application of Theorem 5.1 we shall derive a well-known characterisation of super- and subharmonicity (see $[2,5,7]$ ) relative to a subcone. If $\mathscr{C}$ is a subcone of the locally convex cone $(\mathscr{P}, \mathscr{V})$, an element $a \in \mathscr{P}$ is said to be $\mathscr{C}$-superharmonic in $\mu$ if for every $\nu \in \mathscr{P}^{*}$

$$
\nu(a) \leq \mu(a) \text { holds whenever } \nu(c) \leq \mu(c) \text { for all } c \in \mathscr{C} .
$$

Similarly, $a \in \mathscr{P}$ is said to be $\mathscr{C}$-subharmonic in $\mu$ if for every $v \in \mathscr{P}^{*}$

$$
\nu(a) \geq \mu(a) \text { holds whenever } \quad v(c) \geq \mu(c) \text { for all } c \in \mathscr{C} .
$$

Theorem 5.1 yields.

COROLlaRY 5.3. Let $(\mathscr{P}, \mathscr{V})$ be a locally convex cone, $\mu \in \mathscr{P}^{*}$, and let $\mathscr{C}$ be a subcone of $\mathscr{P}$. An element $a \in \mathscr{P}$ is $\mathscr{C}$-superharmonic in $\mu$ if and only if

$$
\mu(a)=\sup _{v \in \mathscr{V}} \inf \{\mu(c) \mid c \in \mathscr{C}, a \leq c+v\}
$$

Similarly, the element $a \in \mathscr{P}$ such that $\mu(a)$ is finite is $\mathscr{C}$-subharmonic in $\mu$ if and only if

$$
\mu(a)=\inf _{v \in \mathscr{V}} \sup \{\mu(c) \mid c \in \mathscr{C}, c \leq a+v\}
$$

PROOF. We shall argue only the superharmonic case. Let $\mu \in v^{\circ}$, and set $p(a)=$ $\mu(a)$ if $a \in \mathscr{C}$ and $p(a)=+\infty$ otherwise. With $q(a)=-\infty$ for all $0 \neq a \in \mathscr{P}$, the functionals $p$ and $q$ satisfy the hypothesis of Theorem 5.1. For a linear functional $v \in \mathscr{P}^{*}$ we have $q \leq v \leq p$ if and only if $v(c) \leq \mu(c)$ for all $c \in \mathscr{C}$. Following Theorem 5.1 an element $a \in \mathscr{P}$ is therefore $\mathscr{C}$-superharmonic in $\mu$ if and only if

$$
\sup _{v \in \mathscr{V}} \inf \{\mu(c) \mid c \in \mathscr{C}, a \leq c+v\} \leq \mu(a) .
$$

But the reverse inequality holds in any case.

The additional requirement for the second part of Corollary 5.3, namely that $\mu(a)$ should be finite, results from the corresponding condition in Theorem 5.1. It may not be omitted, as the following example will demonstrate. 
EXAMPLE 5.4. Let $\mathscr{P}$ be the cone of all sequences $a=\left(\alpha_{i}\right)_{i \in \mathbb{N}}$ in $\mathbb{R}$ with only finitely many negative elements $\alpha_{i}$, endowed with the pointwise algebraic operations and order. Let the neighbourhood system $\mathscr{V}$ consist of all positive multiples of the element $v=\left(1 / 2^{i-1}\right)_{i \in \mathbb{N}} \in \mathscr{P}$. The linear functional $\mu$ on $\mathscr{P}$ defined by $\mu(a)=(1 / 2) \sum_{i=1}^{\infty} \alpha_{i}$ is clearly continuous with respect to $v$, since $\mu(v)=1$. Now let us consider the subcone $\mathscr{C}$ of $\mathscr{P}$ consisting of all decreasing finite sequences $c=\left(\gamma_{i}\right)_{i \in \mathbb{N}}$, that is $\gamma_{i} \geq \gamma_{i+1} \geq 0$ for all $i \in \mathbb{N}$, and only finitely many of the $\gamma_{i}$ are non-zero. Let $a=(-1,1,1,1, \ldots) \in \mathscr{P}$. Then $\mu(a)=+\infty$, and for all $c=\left(\gamma_{i}\right)_{i \in \mathbb{N}} \in \mathscr{C}$ such that $c \leq a+v$ we have $\gamma_{1}=0$, hence $c=0$. This shows

$$
\inf _{v \in \mathscr{Y}} \sup \{\mu(c) \mid c \in \mathscr{C}, c \leq a+v\}=0<\mu(a) .
$$

But the element $a$ is nevertheless $\mathscr{C}$-subharmonic in $\mu$. Let $\nu \in \mathscr{P}^{*}$ such that $\nu \geq \mu$ on $\mathscr{C}$. Since $v$ is continuous with respect to some positive multiple of $v$, we realise that $\nu(v)$ is finite. For any $n \in \mathbb{N}$ choose $c=\left(\gamma_{i}\right)_{i \in \mathbb{N}} \in \mathscr{C}$ such that $\gamma_{i}=1$ for $i=1, \ldots, 2 n$ and $\gamma_{i}=0$ otherwise. Then $c \leq a+2 v$, hence

$$
n=\mu(c) \leq v(c) \leq v(a)+2 v(v) .
$$

This holds for all $n \in \mathbb{N}$, and since $v(k)<+\infty$, we conclude that $v(a)=$ $\mu(a)=+\infty$.

If the additional assumptions of our Corollary 5.2 apply, then the right hand sides of the conditions in Corollary 5.3 simplify to plain infima respectively suprema. For the superharmonic case this holds true in particular if the neighbourhood system $\mathscr{V}$ is contained in the subcone $\mathscr{C}$ of $\mathscr{P}$. For the subharmonic case, $-\mathscr{V} \subset \mathscr{C}$ suffices.

\section{References}

[1] B. Anger and J. Lembcke, 'Hahn-Banach type theorems for hypolinear functionals', Math. Ann. 209 (1974), 127-151.

[2] H. Bauer, 'Funktionenkegel und Integralungleichungen', in: Bayer. Akad. Wiss. Math.-Natur. Kl. S. - B. 1977 (1978) pp. 53-61.

[3] F. Bonsal, 'Sublinear functionals and ideals in partially ordered vector spaces', Proc. London Math. Soc. 4 (1954), 402-418.

[4] B. Fuchssteiner and W. Lusky, Convex cones, North Holland Math. Stud. 56 (North Holland, Amsterdam, 1981).

[5] K. Keimel and W. Roth, Ordered cones and approximation, Lecture Notes in Math. 1517 (Springer, Berlin, 1992).

[6] H. König, 'Sublineare Funktionale', Arch. Math. 23 (1972), 500-508.

[7] J. Lembcke, 'Note zu "Funktionenkegel und Integralungleichungen" von H. Bauer', in: Bayer. Akad. Wiss. Math.-Natur. Kl. S.-B. 1977 (1978) pp. 139-142.

[8] L. Nachbin, Topology and order (Van Nostrand, Princeton, 1965). 
[9] J. M. A. M van Neerven, 'Hahn-Banach type theorems for adjoint semigroups', Math. Ann. 387 (1990), 63-71.

[10] P. Plappert, 'A sandwich theorem for monotone additive functions', Semigroup Forum 51 (1995), 347-355.

[11] W. Roth, 'A combined approach to the fundamental theorems for normed spaces', Bulletin Acad. Sinica 22 (1994), 83-89.

Department of Mathematics

Faculty of Science

University Brunei Darussalam

Bandar Seri Begawan 2028

Brunei Darussalam

e-mail: roth@ubd.edu.bn 